\title{
First report of urinary tract infection caused by Comamonas kerstersii in a goat
}

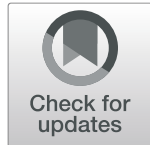

\author{
Silvia Pavone", Roberto Rinoldo, Elisa Albini, Alessandro Fiorucci, Biagio Caponi, Anna Fratto, Elisabetta Manuali, \\ Paola Papa and Chiara Francesca Magistrali
}

\begin{abstract}
Background: Comamonas kerstersii is rarely associated with infections in humans and has never been reported in animals until now.

Case presentation: Herein, we describe a case of urinary tract infection caused by C. kerstersii in a young goat. A seven-month-old male goat showed lethargy, generalised weakness and anorexia and in the last hours before its death, severe depression, slight abdominal distention, ruminal stasis, and sternal recumbency. Grossly, multifocal haemorrhages in different organs and tissues, subcutaneous oedema and hydrocele, serous fluid with scattered fibrin deposition on the serosa of the abdominal organs and severe pyelonephritis with multifocal renal infarction were detected. Histopathological examination confirmed severe chronic active pyelonephritis with renal infarcts, multi-organ vasculitis and thrombosis suggestive of an infectious diseases of bacterial origin. The bacterium was identified using routine methods, matrix assisted laser desorption/ionisation time-of-flight mass spectrometry (MALDI-TOF-MS), and sequencing of the gyrB gene.

Conclusions: To the best of our knowledge, this is the first report of $C$. kerstersii infection in animals (goat). Our findings support the possibility of $C$. kerstersii isolation from extraintestinal sites and suggest this organism as a possible cause of urinary tract infection.
\end{abstract}

Keywords: Comamonas kerstersii, Emerging pathogen, Goat, Pyelonephritis, Urinary tract infection

\section{Background}

Comamonas kerstersii is a gram-negative, nonfermenting, oxidase- and catalase-positive motile bacterium first described in 2003 [1]. The genus Comamonas originally contained C. terrigena, C. testosteroni (previously Pseudomonas testosteroni), and C. acidovorans (previously P. acidovorans) [2]. Now, Comamonas genus contains 17 species including C. terrigena, C. aquatica, C. kerstersii, C. testosteroni, C. denitrificans, C. nitrativorans, C. koreensis, and others [3]. Although they are ubiquitously distributed in the environment (soil and water), Comamonas sp. are rarely associated with

\footnotetext{
* Correspondence: s.pavone@izsum.it

Istituto Zooprofilattico Sperimentale dell'Umbria e delle Marche 'Togo Rosati', Via G. Salvemini, 1, 06126 Perugia, Italy
}

infections in humans and have never been reported in animals until now. In particular, $C$. kerstersii has recently been associated with human invasive infections, such as abdominal and urinary tract infections, and bacteraemia [4-8].

Herein, we describe a case of urinary tract infection caused by Comamonas kerstersii in a young goat. To the best of our knowledge, this is the first report of C. kerstersii in animals.

\section{Case presentation}

A post mortem examination was performed on a sevenmonth-old male goat with a history of lethargy, generalised weakness and anorexia and in the hours preceding its death, severe depression, slight abdominal distention, ruminal stasis, and sternal recumbence. The animal

(c) The Author(s). 2021 Open Access This article is licensed under a Creative Commons Attribution 4.0 International License, which permits use, sharing, adaptation, distribution and reproduction in any medium or format, as long as you give appropriate credit to the original author(s) and the source, provide a link to the Creative Commons licence, and indicate if changes were made. The images or other third party material in this article are included in the article's Creative Commons licence, unless indicated otherwise in a credit line to the material. If material is not included in the article's Creative Commons licence and your intended use is not permitted by statutory regulation or exceeds the permitted use, you will need to obtain permission directly from the copyright holder. To view a copy of this licence, visit http://creativecommons.org/licenses/by/4.0/ The Creative Commons Public Domain Dedication waiver (http://creativecommons.org/publicdomain/zero/1.0/) applies to the data made available in this article, unless otherwise stated in a credit line to the data. 
came from a farm in central Italy consisting of twenty adult goats (two males and eighteen females).

Histologic samples of organs with pathologic changes were collected and the tissues were fixed in $10 \%$ neutral buffered formalin, embedded in paraffin wax, sectioned at $5 \mu \mathrm{m}$ and stained with haematoxylin and eosin.

Spleen samples were collected for molecular analysis of the Border disease virus (BDV). Molecular genetic investigation was based on the $5^{\prime}$ untranslated region (UTR) coding. Intestinal, pulmonary, hepatic, and renal specimens were aseptically collected for bacteriological examination. Moreover, peritoneal swabs and urine samples obtained by cystocentesis were tested. The samples were spread plated on MacConkey agar, mannitol salt agar and blood agar plates for aerobic incubation at $37{ }^{\circ} \mathrm{C}$ for 3 days. Suspected colonies were identified using a MALDI-TOF MS instrument (Bruker Daltonics, Bremen, Germany) with Microflex LT Smart Biotyper with FlexControl Biotyper 3.4 software (Bruker Daltonics, Bremen, Germany). Identification was confirmed by sequencing the $\operatorname{gyr} B$ encoding for the $B$ subunit of the DNA gyrase, using the primers described by Tayeb et al. [9]. Antibiotic susceptibility testing was performed using a commercially available MIC plate (COMPAN2F, Thermo Fisher Scientific, Waltham, USA). The performance of MIC testing was verified using E. coli ATCC 25, 922 as a control strain, as recommended by Clinical \& Laboratory Standards Institute (CLSI) [10]. The minimal inhibitory concentration (MIC) of ampicillin was further evaluated using a Liofilchem MIC strip (Liofilchem, Teramo, Italy). Briefly, a 1 McFarland standard-matched suspension from a 24-h pure culture on Brucella blood agar supplemented with $5 \%$ horse red blood cells (Brucella agar, hemin, Sigma-Aldrich, Milan, Italy) was distributed on a Brucella blood agar plate with the MIC strip. The culture plate was incubated at $37^{\circ} \mathrm{C}$ for 24 -h and MIC determination was performed according to the manufacturer's instructions. The CLSI breakpoints for 'other non-Enterobacterales' [10], when available, were used to interpret the MIC results as susceptible, intermediate and resistant. The MIC values for amoxicillin + clavulanic acid, ampicillin, cefazolin, penicillin, and ticarcillin were interpreted using pharmacokinetic/pharmacodynamic (PK/PD) values, according to the European Committee on Antimicrobial Susceptibility Testing (EUCAST) recommendations [11]. We investigated the presence of $\operatorname{str} A, \operatorname{str} B$, tet( $A)$, sul1, sul2, and bla $a_{\mathrm{OXA}}$ $-1 / 4 / 30$, which have already been described in C. kerstersii [12]. Furthermore, we assessed the presence of other genes coding for resistance to beta-lactams and aminoglycosides using polymerase chain reaction (PCR) tests targeting aac(6)-Ib-cr, aadA, bla $a_{\mathrm{TEM}-1 / 2}, \quad b l a_{\mathrm{SHV}-1}$, $b l a_{\mathrm{CTX}-\mathrm{M}-1}, b l a_{\mathrm{CTX}-\mathrm{M}-2}, b l a_{\mathrm{CTX}-\mathrm{M}-9}, b l a_{\mathrm{CTX}-\mathrm{M}-8}$ and $b l a_{\mathrm{CTX}-\mathrm{M}-25}$, as previously described [13-20].
For anaerobic incubation, the samples were spread also on a blood agar plate placed in an anaerobic jar with AnaeroGen reagent (Thermo Fisher Scientific, Waltham, USA) and incubated at $37{ }^{\circ} \mathrm{C}$ for $18-24 \mathrm{~h}$. Incubation was prolonged for two more days if no colonies appeared at $24 \mathrm{~h}$.

Coprological examination was carried out using the FLOTAC technique on five grams of feces.

At necropsy, the goat appeared in good condition (body condition score $=3$ ) and in a good conservation status, but was dehydrated. Conjunctival, subcutaneous, and muscular haemorrhages associated with subcutaneous oedema and hydrocele were found. Within the abdominal cavity, there was moderate serous fluid with scattered fibrin deposition on the serosa of the abomasum, liver, and intestinal loops. Petechiae and ecchymosis were detected in the perirenal adipose tissue (Fig. 1a). The kidneys were swollen and markedly congested. On the cut surface, the renal cortex of the left kidney showed discolouration with pink-gray areas alternating with hemorrhagic wedge-shaped areas (renal infarction) (Fig. 1b). The pelvis showed yellowish and grey areas, and was oedematous and grossly free of obstruction. The contralateral kidney showed marked congestion and haemorrhages. No obstruction or apparent lesions on the mucosa of the ureters, bladder, or urethra were detected. Slightly cloudy and dark urine samples were observed. Congestion of the liver and catarrhal enteritis was also detected. In the thoracic cavity, the lungs were patchily congested and haemorrhagic; endocardial and epicardial haemorrhages were observed. No other macroscopic lesions were observed.

Histological examination of the left kidney showed large areas of cortical necrosis of glomeruli and tubules surrounded by tubule-interstitial infiltration with neutrophils (Fig. 2a and b). Marked congestion and dilation of the glomerular capillary loop with glomerular collapse and accumulation of fibrin and cellular debris in the Bowman's space were observed (Fig. 2b). Extensive necrotic processes associated with abundant interstitial and intratubular neutrophilic infiltrates were detected in the renal pelvis; multifocal areas of interstitial fibrosis with scattered lymphocytes and plasma cells (chronic inflammation) can be found in the renal papillae. Vasculitis, fibrinoid necrosis, and thrombosis of the vessels of the renal capsule associated with severe oedema were detected. In the right renal tissue, there was glomerular collapse with an accumulation of cellular debris in the Bowman's space, scattered necrotic tubules (Fig. 2c), and fibrosing, necrotizing, and lymphoplasmacellular and suppurative pyelitis (chronic-active inflammation). Severe vasculitis and fibrinoid necrosis of small to medium arteries of the penile urethra (Fig. 2d) associated with degenerative changes, and attenuation of the transitional 


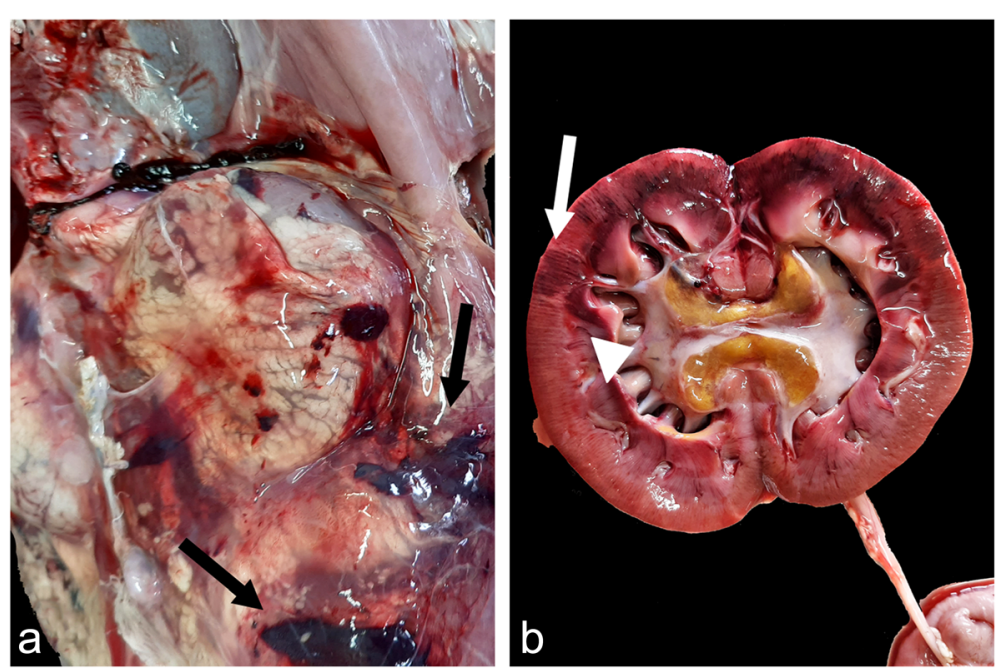

Fig. 1 Photographs of gross lesions in the urinary tract of goat. a Oedema and hemorrhages (arrows) of perirenal adipose tissue in the left renal lodge. b Left kidney. Cortical discoloration with hemorrhagic wedge-shaped area (arrow) and pelvis characterized by greyish and yellowish tissue

epithelium of ureters, and marked interstitial oedema of peripheral connective and adipose tissue were observed. On the other hand, histological examination of the intestine showed moderate, subacute, diffuse lymphocytic and eosinophilic enteritis, whereas the lungs showed alveolar oedema, severe congestion of pulmonary capillaries and multifocal hemorrhages. Overall, the lesions were consistent with severe chronic active pyelonephritis with renal infarcts, multi-organ vasculitis, and thrombosis suggestive of infectious diseases of bacterial origin.
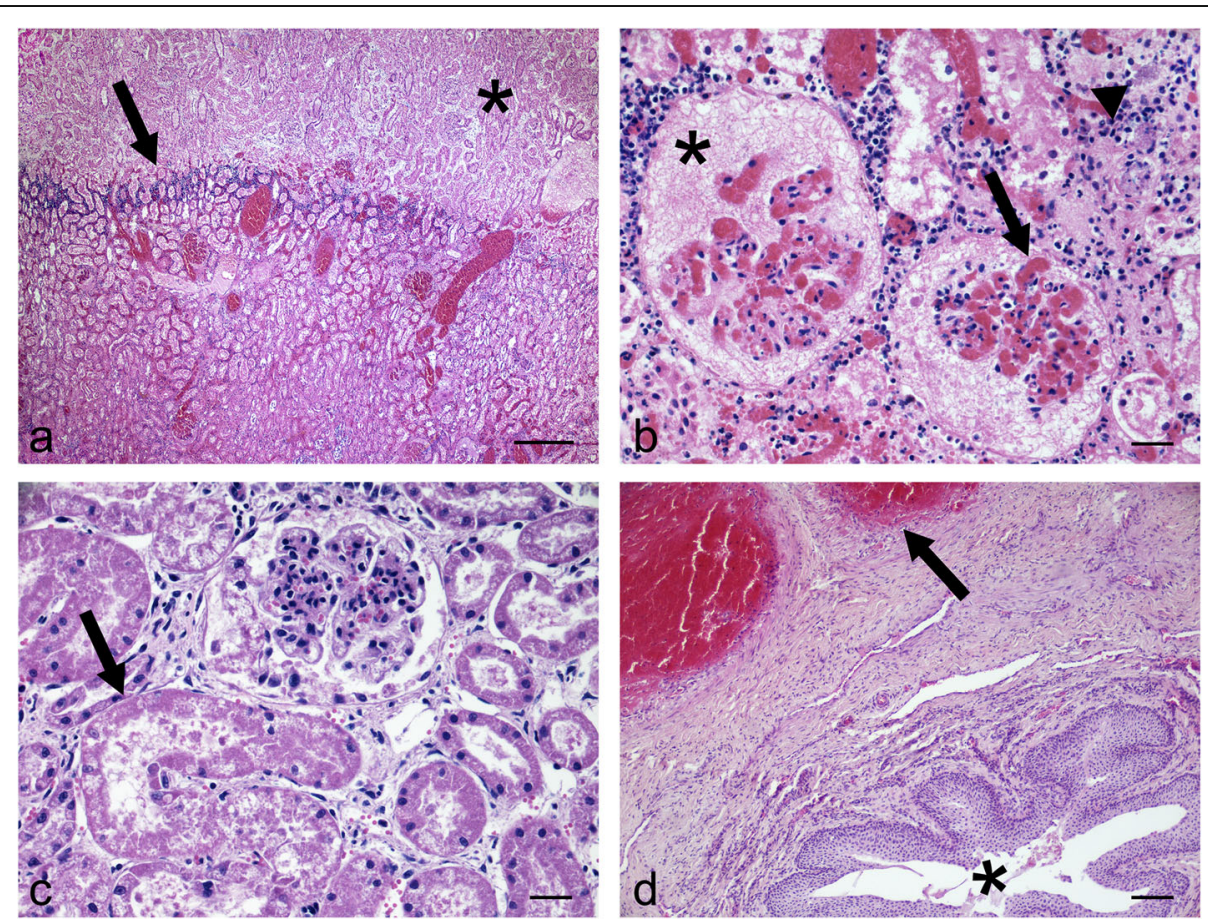

Fig. 2 Histological changes in the urinary tract of goat. a Left kidney characterized by extensive cortical coagulation necrosis (asterisk) bounded by neutrophilic tubule-interstitial infiltration (arrow). Marked and diffuse vascular congestion. (Hematoxylin and eosin, Bar = 200). b Left kidney showed marked congestion and dilation of glomerular capillary loop (arrow) with glomerular collapse and accumulation of fibrin in Bowman's space (asterisk). Interstitial neutrophilic inflammatory infiltrate and scattered foci of mineralization (arrowhead). (Hematoxylin and eosin, Bar = 25). c Right kidney showed glomerular collapse with accumulation of cellular debris in Bowman's space and necrosis of renal tubules (arrow). (Hematoxylin and eosin, Bar =25). d Vasculitis (arrow) of medium arteries of penile urethra (asterisk). (Hematoxylin and eosin, Bar = 100) 
The bacteriological examinations showed negative results in pulmonary, intestinal and hepatic specimens, and from peritoneal swabs. From the renal specimen and the urine, we observed the growth of a smooth, light-grey, non-haemolytic colony in pure culture. The colony was identified as Comamonas kerstersii by MALDI-TOF MS with a high-confidence identification spectral score of (2.22) and high consistency interpretation (A), since the same species was identified as the second- and third-best match. Other species within the genus, C. testosteroni and C. aquatica, resulted in low identification score, 1.42 and 1.32, respectively. An alignment search for the $g y r B$ sequence using the Basic Local Alignment Search Tool (BLAST) on the NCBI website revealed $99.07 \%$ identity with the $\operatorname{gyr} B$ sequence of the $C$. kerstersii strain 8943 sequence (accession number CP020121.1), confirming the previous MALDI-TOF MS identification. The sequence of the $g y r B$ gene of the $C$. kestersii described in this manuscript has been submitted to NCBI GenBank (GenBank accession no. MW349112).

The MIC values of the control strain were in accordance with the reference values reported by the CLSI [10]. For the C. kerstesii isolate, the following MICs (mg/L) were determined: amikacin, 8; amoxicillin + clavulanic acid, 0.25/0.12; ampicillin, $\geq 1$; cephalothin, $\leq 2$; cefazolin, $\leq 1$; cefovecin, $\leq 0.25$; cefoxitin, $\leq 2$; cefpodixime, $\leq 2$; ceftiofur, $\leq 0.25$; chloramphenicol, $\leq 4$; doxycycline $\leq 2$; enrofloxacin, $\leq 0.25$; gentamicin, 2 ; imipenem, $\leq 1$; marbofloxacin, $\leq 0.25$; penicillin, 2 ; ticarcillin, $\leq 8$; ticarcillin + clavulanic acid, $\leq 8 / 2$; trimethoprim + sulphamethoxazole, $\leq 0.5 / 9.5$. According to the CLSI breakpoints [10], the isolate was classified as susceptible to amikacin, chloramphenicol, doxycycline, gentamicin, imipenem, ticarcillin + clavulanic acid and trimethoprim + sulphamethoxazole. Based on the PK/PD values [11], the isolate was not susceptible to penicillin and ampicillin, but susceptible to amoxicillin + clavulanic acid, cefazolin, and ticarcillin. No breakpoints were available for the other tested molecules from the same source. The strain tested positive for strA and strB genes that confer resistance to streptomycin, whereas other antibiotic-resistance genes were not found.

Copromicroscopic examination revealed 2064 coccidian oocysts per gram of faeces (o.p.g). On the other hand, negative results were obtained from molecular analysis of the BDV.

\section{Discussion and conclusions}

To date, infections caused by Comamonas kerstersii have been reported only in humans. This infection is often associated with peritonitis resulting from the presence of a perforated appendix, diverticulosis, pelvic peritonitis, and bacteraemia [4-7]. More recently, an unusual case of urinary tract infection in a 5-year-old girl was reported [8].

Herein, we reported a case of chronic-active necrotising and suppurative pyelonephritis caused by Comamonas kerstersii isolated from both renal specimens and from urine, and identified with the use of MALDI-TOF MS. It is well known that the use of MALDI-TOF MS for routine bacterial identification has revolutionised microbiology, allowing the identification of infectious agents efficiently and quickly [8]. The growing use of this method in veterinary medicine is likely to allow to identify an increasing number of pathogens considered rare or never detected in animals.

The increase in Comamonas kerstersii isolation frequency from human infections in the literature and now the isolation in urinary tract infection in goat identifies it as an emerging pathogen. Since translocation from the digestive tract seems to be a predominant cause of infections by $C$. kerstersii [21], we looked for this microorganism in the intestinal content, but obtained negative results. Therefore, and in view of the highlighted renal histological pattern (medullary changes seemed to become chronic while the cortical lesions were yet in an acute phase), the ascending path represents the most likely route of infection in this case.

As previously suggested, Comamonas kerstersii could be an opportunistic pathogen or commensal in the digestive tract and appendix in humans [22]. In this case, the presence of $C$. kerstersii as the only microorganism isolated from the urinary tract of goat, with no apparent known cause of immunodeficiency disorders points to it as a primary pathogen; however, a severe coccidiosis found in the present goat may represent a cause of debilitation and potential predisposing factor for opportunistic bacterial infections.

According to CLSI breakpoints for 'other nonEnterobacterales' [10], our strain showed susceptibility to antimicrobials, but this finding should be interpreted with caution since no specific breakpoints for the Comamonas genus are available. We observed high MIC values for two aminoglycosides, gentamicin and amikacin, and the presence of two genes encoding resistance to streptomycin, another member of the same antibiotic class. The presence of these genes was detected in the same species by Jiang et al. [12]. Our isolate was also classified as non-susceptible to ampicillin and penicillin according to the EUCAST PK/PD breakpoints. There is a paucity of data on the susceptibility of $C$. kerstersii to antibiotics in the literature, although other authors have reported the susceptibility of $C$. kerstersii isolates to ampicillin $[4,7]$, suggesting that $C$. kerstersii is not naturally resistant to ampicillin. Further research is needed 
to investigate the genetic background of resistance to $\beta$ lactamase in this species. Indeed, the presence of class A $\beta$-lactamase has already been described in another member of the genus, $C$. testosteronii [23].

In conclusion, this report describes the first case of Comamonas kerstersii infection in animals (goat). Our findings support the possibility of $C$. kerstersii isolation from extraintestinal sites and suggest this organism as a possible cause of urinary tract infection.

\section{Acknowledgements}

Not applicable.

\section{Authors' contributions}

SP performed histopathology and drafted the report, RR performed antibiotic susceptibility testing, EA performed identification of colonies by MALDI-TOF method and molecular investigations and sequencing, AF performed bacteriological investigation, BC performed pathology, AF performed histology tissue processing, EM interpreted the data and edited text, PP examined the case and finalized report, CFM interpreted the data and substantively revised the work. All authors have read and approved the manuscript.

\section{Funding}

This work has not been founded.

\section{Availability of data and materials}

The datasets used and analyzed during the current study are available from the corresponding author on reasonable request.

\section{Declarations}

\section{Ethics approval and consent to participate}

All examinations were performed with written informed owner's consent according to ethical guidelines of the Istituto Zooprofilattico Sperimentale dell'Umbria e delle Marche 'Togo Rosati'.

\section{Consent for publication}

Informed written consent to publish all given information was obtained from the owner of the presented goat.

\section{Competing interests}

The authors declare that the study was conducted in the absence of any commercial or financial relationships that could be construed as a potential conflict of interest.

Received: 14 January 2021 Accepted: 16 March 2021

Published online: 25 March 2021

\section{References}

1. Wauters G, De Baere T, Willems A, Falsen E, Vaneechoutte M. Description of Comamonas aquatica comb. nov. and Comamonas kerstersii sp. nov. for two subgroups of Comamonas terrigena and emended description of Comamonas terrigena. Int J Syst Evol Microbiol. 2003;53:859-62.

2. De Vos P, Kersters K, Falsen E, Pot B, Gillis M, Segers P, De Ley J. Comamonas Davis and Park 1962 gen. nov., nom. rev. emend., and Comamonas terrigena Hugh 1962 sp. nov. Int J Syst Bacteriol. 1985;35:44353.

3. Nakipoglu Y, Erturan Z, Buyukbaba-Boral O, Aksozek A, Aydin S, Derbentli S. Evaluation of the contaminant organisms of humidifier reservoir water and investigation of the source of contamination in a university hospital in Turkey. Am J Infect Control. 2005;33:62-3.

4. Almuzara MN, Cittadini R, Ocampo CV, Bakai R, Traglia G, Ramirez MS, del Castillo M, Vay CA. Intra-abdominal infections due to Comamonas kerstersii. J Clin Microbiol. 2013;51:1998-2000.

5. Opota O, Ney B, Zanetti G, Jaton K, Greub G. Prod'hom G. Bacteremia caused by Comamonas kerstersii in a patient with diverticulosis. J Clin Microbiol. 2014;52:1009-12.

6. Biswas J, Fitchett J, O'Hara G. Comamonas kerstersii and the perforated appendix. J Clin Microbiol. 2014;52:3134.
7. Almuzara M, Barberis C, Veiga F, Bakai R, Cittadini R, Ocampo VC, Serena MA, Cohen E, Ramirez MS, Famiglietti A, Stecher D, del Castillo M, Vay C. Unusual presentations of Comamonas kerstersii infection. New Microbes New Infect. 2017;19:91-5.

8. Almuzara M, Cittadini R, Estraviz ML, Ellis A, Vay C. First report of Comamonas kerstersii causing urinary tract infection. New Microbes New Infect. 2018;24:4-7.

9. Tayeb LA, Lefevre M, Passet V, Diancourt L, Brisse S, Grimont PA. Comparative phylogenies of Burkholderia, Ralstonia, Comamonas, Brevundimonas and related organisms derived from rpoB, gyrB and rrs gene sequences. Res Microbiol. 2008;159:169-77.

10. Clinical and Laboratory Standard Institute (CLSI). Performance Standards for Antimicrobial Susceptibility Testing. 30th ed. CLSI supplement M100. CLSI. Wayne, PA 19087 USA, 2020.

11. EUCAST, European Committee of Antimicrobial Susceptibility testing. https://eucast.org/. Accessed 12th February, 2021.

12. Jiang $X$, Liu W, Zheng B. Complete genome sequencing of Comamonas kerstersii 8943, a causative agent for peritonitis. Sci Data. 2018;5:180222.

13. Park CH, Robicsek A, Jacoby GA, Sahm D, Hooper DC. Prevalence in the United States of aac $\left(6^{\prime}\right)-\mathrm{lb}$-cr encoding a ciprofloxacin-modifying enzyme. Antimicrob Agents Chemother. 2006;50:3953-5.

14. Pei R, Kim SC, Carlson KH, Pruden A. Effect of river landscape on the sediment concentrations of antibiotics and corresponding antibiotic resistance genes (ARG). Water Res. 2006;40:2427-35.

15. Dallenne C, Da Costa A, Decré D, Favier C, Arlet G. Development of a set of multiplex PCR assays for the detection of genes encoding important betalactamases in Enterobacteriaceae. J Antimicrob Chemother. 2010;65:490-5.

16. Walsh F, Ingenfeld A, Zampicolli M, Hilber-Bodmer M, Frey JE, Duffy B. Realtime PCR methods for quantitative monitoring of streptomycin and tetracycline resistance genes in agricultural ecosystems. J Microbiol Methods. 2011;86:150-5.

17. Guo MT, Yuan QB, Yang J. Ultraviolet reduction of erythromycin and tetracycline resistant heterotrophic bacteria and their resistance genes in municipal wastewater. Chemosphere. 2013;93:2864-8.

18. Eitel Z, Sóki J, Urbán E, Nagy E, ESCMID Study Group on Anaerobic Infection. The prevalence of antibiotic resistance genes in Bacteroides fragilis group strains isolated in different European countries. Anaerobe. 2013;21:43-9.

19. He LY, Liu YS, Su HC, Zhao JL, Liu SS, Chen J, Liu WR, Ying GG. Dissemination of antibiotic resistance genes in representative broiler feedlots environments: identification of indicator ARGs and correlations with environmental variables. Environ Sci Technol. 2014;48:13120-9.

20. Pholwat S, Liu J, Taniuchi M, Chinli R, Pongpan T, Thaipisutikul I, Ratanakorn P, Platts-Mills JA, Fleece M, Stroup S, Gratz J, Mduma E, Mujaga B, Walongo T, Nshama R, Kimathi C, Foongladda S, Houpt ER. Genotypic antimicrobial resistance assays for use on $\mathrm{E}$. coli isolates and stool specimens. PLoS One. 2019;14:e0216747

21. Hagiya $H$, Murase $T$, Sugiyama J, Kuroe $Y$, Nojima $H$, Naito H, Hagioka $S$, Morimoto N. Delftia acidovorans bacteremia caused by bacterial translocation after organophosphorus poisoning in an immunocompetent adult patient. J Infect Chemother. 2013;19:338-41.

22. Zhou Y, Ma H, Dong Z, Shen M. Comamonas kerstersii bacteremia in a patient with acute perforated appendicitis. Medicine. 2018:97:13(e9296).

23. Zhuang W, Liu H, Li J, Chen L, Wang G. Regulation of Class A $\beta$-Lactamase CzoA by CzoR and IsCR in Comamonas testosteroni S44. Front Microbiol. 2017:8:2573

\section{Publisher's Note}

Springer Nature remains neutral with regard to jurisdictional claims in published maps and institutional affiliations. 